Cronfa - Swansea University Open Access Repository

This is an author produced version of a paper published in:

Nanotechnology

Cronfa URL for this paper:

http://cronfa.swan.ac.uk/Record/cronfa38951

\title{
Paper:
}

Barnett, C., Jackson, G., Jones, D., Lewis, A., Welsby, K., Evans, J., McGettrick, J., Watson, T., Maffeis, T., et. al. (2018). Investigation into the effects of surface stripping $\mathrm{ZnO}$ nanosheets. Nanotechnology, 29(16), 165701 http://dx.doi.org/10.1088/1361-6528/aaae5c

This item is brought to you by Swansea University. Any person downloading material is agreeing to abide by the terms of the repository licence. Copies of full text items may be used or reproduced in any format or medium, without prior permission for personal research or study, educational or non-commercial purposes only. The copyright for any work remains with the original author unless otherwise specified. The full-text must not be sold in any format or medium without the formal permission of the copyright holder.

Permission for multiple reproductions should be obtained from the original author.

Authors are personally responsible for adhering to copyright and publisher restrictions when uploading content to the repository. 


\section{Investigation into the Effects of Surface Stripping ZnO Nanosheets}

Chris J. Barnett*1, Georgina Jackson ${ }^{2}$, Daniel R. Jones ${ }^{1}$, Aled R. Lewis ${ }^{3}$, Kathryn Welsby ${ }^{2}$, Jon E. Evans ${ }^{3}$, James D. McGettrick ${ }^{3}$, Trystan Watson ${ }^{3}$, Thierry G. G. Maffeis ${ }^{3}$, Peter R. Dunstan $^{2}$, Andrew R. Barron ${ }^{1,4,5}$ and Richard J. Cobley ${ }^{3}$

1 Energy Safety Research Institute, Swansea University, Bay Campus, Swansea SA1 8EN, U.K.

2 Department of Physics, College of Science, Swansea University, Singleton Park, Swansea SA2 8PP, U.K.

3 College of Engineering, Swansea University, Bay Campus, Swansea SA1 8EN, U.K.

4 Department of Chemistry, Rice University, Houston, Texas 77005, U.S.A.

5 Department of Materials Science and Nanoengineering, Rice University, Houston, Texas 77005, U.S.A.

c.j.barnett@swansea.ac.uk, 741330@swansea.ac.uk, d.r.jones@swansea.ac.uk, 557952@swansea.ac.uk, k.a.welsby@swansea.ac.uk, j.e.evans@swansea.ac.uk, j.d.mcgettrick@swansea.ac.uk, t.m.watson@swansea.ac.uk, t.g.g.maffeis@swansea.ac.uk, p.r.dunstan@swansea.ac.uk, a.r.barron@swansea.ac.uk, richard.j.cobley@swansea.ac.uk.

Keywords: ZnO, Nanosheets, Argon, XPS, Ohmic, Schottky, Photoluminescence, Conduction 


\begin{abstract}
$\mathrm{ZnO}$ nanosheets are polycrystalline nanostructures that are used in devices including solar cells and gas sensors. However, for efficient and reproducible device operation and contact behaviour the conductivity characteristics must be controlled and surface contaminants removed. Here we use low doses of argon bombardment to remove surface contamination and make reproducible lower resistance contacts. Higher doses strip the surface of the nanosheets altering the contact type from near-ohmic to rectifying by removing the donortype defects, which PL shows to be concentrated in the near-surface. Controlled doses of argon treatments allow nanosheets to be customised for device formation.
\end{abstract}

\title{
Introduction
}

Zinc Oxide $(\mathrm{ZnO})$ is a wide band gap $(3.37 \mathrm{eV})$ metal oxide piezoelectric semiconductor that has the ability to be formed in numerous nanostructures including nanowires, nanorods and nanosheets [1-3]. Due to their high surface area to volume ratio, polycrystalline $\mathrm{ZnO}$ nanosheets have been shown to have potential practical application in gas-sensing devices and dye-sensitised solar cells [4-6].

These devices require surface contamination to be removed in order to create a consistent contact. One method of doing this involves annealing the nanosheets; however, previous work has shown that vacuum annealing causes changes in the polycrystalline structure and to the defect chemistry, which affects the conduction characteristics of the nanosheets [7].

Other commonly used methods to clean nanostructures are plasma treatments and argon bombardment [8-10]. Argon plasma treatment has been observed to increase the mobility, carrier concentration and conductivity of $\mathrm{ZnO}$ nanowires [11] and has been used by Dulub et al. [12] to clean single crystal $\mathrm{ZnO}$ in order to carry out scanning tunnelling microscopy (STM). Our previous work found that argon bombardment using $0.3 \mathrm{kV}$ for 20 minutes on $\mathrm{ZnO}$ nanowires removed surface contamination resulting in a more consistent contact and a three orders of magnitude drop in resistance due to the reduced contact resistance [13]. It was also found that higher powered argon bombardment (1-2.5 kV for 20 minutes) stripped the surface resulting in a reduction in the number of defects observed using photoluminescence (PL). This indicated that the reduction in donor states caused the formation of a more rectifying contact and suggested that the defects are situated at the near- 
surface of $\mathrm{ZnO}$ nanorods. It is not known whether the defects of polycrystalline $\mathrm{ZnO}$ are situated in the near-surface and can be stripped using argon bombardment in order to control the conductivity and contact type.

Here we investigate the effects of argon bombardment on the conduction characteristics and chemistry of polycrystalline $\mathrm{ZnO}$ nanosheets using scanning electron microscope (SEM), Xray photoelectron spectroscopy (XPS), two-point probe and PL.

\section{Method}

Layered basic zinc acetate (LBZA) nanosheets were synthesised in an $800 \mathrm{~W}$ commercial microwave in a $500 \mathrm{ml}$ solution of $0.1 \mathrm{M}$ zinc acetate dihydrate $\left(\mathrm{Zn}\left(\mathrm{CH}_{3} \mathrm{COO}\right)_{2} \cdot 2 \mathrm{H}_{2} \mathrm{O}\right)$ and $0.04 \mathrm{M}$ hexamethylenetetramine (HMTA, $\left.\left(\mathrm{CH}_{2}\right)_{6} \mathrm{~N}_{4}\right)$ for 6 mins [6]. The solution was then centrifuged, the supernatant removed and the residue re-suspended in DI water. The LBZA nanosheets were then deposited on n-type (100) silicon and annealed in air at $400{ }^{\circ} \mathrm{C}$ to thermally decompose the LBZA into $\mathrm{ZnO}$. Thirteen samples were made in this way.

An Omicron LT nanoprobe equipped with a PSP ISIS 3000 ion gun was used to argon bombard the samples. One sample was loaded into the system but not treated with argon to act as a control, hereafter known as the 'before' sample. The remaining 12 samples were subjected to various argon treatments at room temperature using energies of $0.3,1.0$ and 2.0 $\mathrm{keV}$ for time lengths ranging between 5 and 20 minutes.

Nanoscale two-point probe measurements were performed using tungsten probes that had been annealed to reduce probe oxide contamination $[14,15]$ and approached using a method to ensure minimal compressive strain at the point of contact providing intrinsic characterisation [16]. Five I-V repeat sweeps were performed between potential differences of $-2 \mathrm{~V}$ and $2 \mathrm{~V}$ on randomly selected nanosheets and the probe separation was varied. All measurements were carried out at room temperature.

Surface chemical characterisation was carried out using a Kratos AXIS Supra XPS, Hitachi S4800 SEM and PL measurements obtained through use of a 325-nm wavelength He-Cd laser and an Ocean Optics USB2000+ spectrometer. For the XPS, three areas of the samples were scanned using a monochromatic $\mathrm{Al} \mathrm{K}_{\alpha} \mathrm{X}$-ray source and a charge neutraliser, with spectra measured at a pass energy of $20 \mathrm{eV}$. In the case of the PL measurements, three 
spectra were acquired at room temperature from different areas of the sample and then normalised and averaged.

\section{Results and Discussion}

The synthesised $\mathrm{ZnO}$ nanosheets were of rectangular form with lengths typically ranging from 1 to $20 \mu \mathrm{m}$ and widths of 1 to $15 \mu \mathrm{m}$, which is in general agreement with our previous results [7]. Figure 1 shows a SEM image of the grain structure of a typical $\mathrm{ZnO}$ nanosheet before argon bombardment, Figure S1 in the supplemental information shows the nanosheet coverage of the sample. The average grain size was $5 \mathrm{~nm}$ and the surface consisted of voids and uneven features.

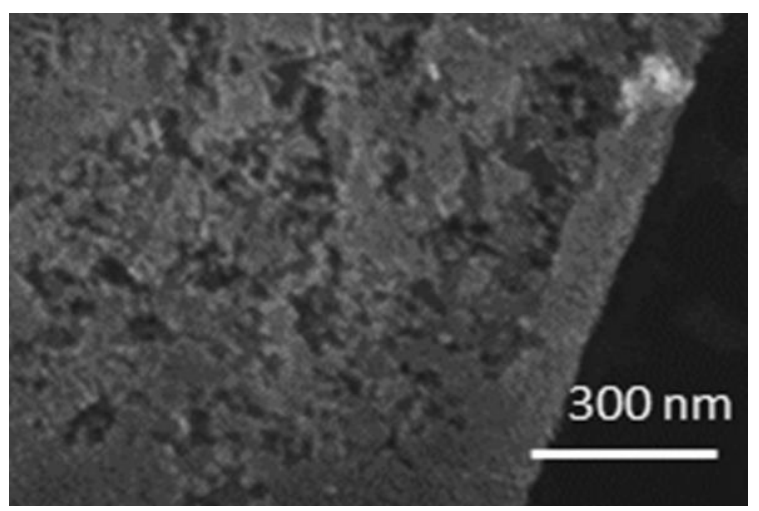

Figure 1: SEM image of $\mathrm{ZnO}$ nanosheet before argon bombardment.

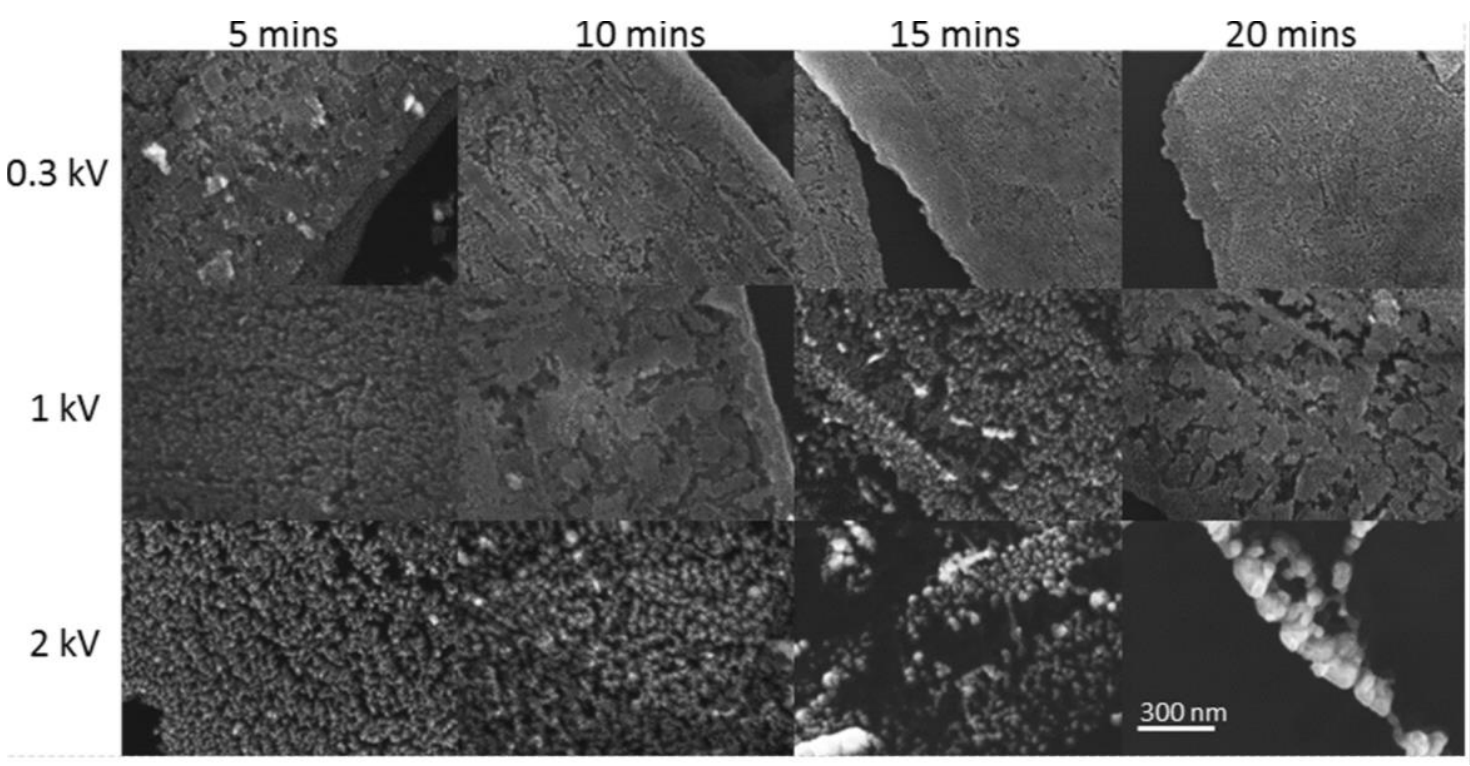


Figure 2: SEM images of $\mathrm{ZnO}$ nanosheets after argon bombardment of $0.3 \mathrm{kV}, 1 \mathrm{kV}$ and $2 \mathrm{kV}$ for time durations 5, 10, 15 and 20 minutes.

Figure 2 shows SEM images of samples subjected to argon bombardment. Argon bombardment at $0.3 \mathrm{kV}$ caused no observable change to the structure of the $\mathrm{ZnO}$ nanosheets while bombardment at $1 \mathrm{kV}$ caused observable damage to the $\mathrm{ZnO}$ nanosheets by stripping the surface causing the silicon substrate to be visible under the nanosheets. Bombardment at 2 $\mathrm{kV}$ also caused observable damage to the nanosheets, with bombardment of duration greater than 15 mins resulting in the nanosheets being almost completely destroyed.

XPS scans were recorded to assess whether the argon bombardment had had an effect on the surface chemistry, including a survey spectrum and detailed spectra of the $\mathrm{Zn} 2 \mathrm{p}, \mathrm{Zn} 3 \mathrm{p}, \mathrm{O}$ 1s, Ar 2p and C 1s core levels and Zn LMMA. Figure 3 shows the changes in the survey scan, $\mathrm{Zn} 2 \mathrm{p}$ core level and the Zn LMMA scans following argon bombardment at different powers and times, and the $\mathrm{O} 1 \mathrm{~s}$ fit. The survey spectrum shows a peak at $99.4 \mathrm{eV}$, which is associated with Si from the silicon dioxide substrate. Analysis of the $\mathrm{C}$ 1s peak revealed that the quantity of carbon varied randomly with bombardment dose, which we attribute to the random distribution of carbon throughout the $\mathrm{ZnO}$ nanosheet structure resulting from the incomplete oxidation of zinc acetate during the annealing. This meant that analysis of carbon contamination removal due to the argon bombardment was not possible. 

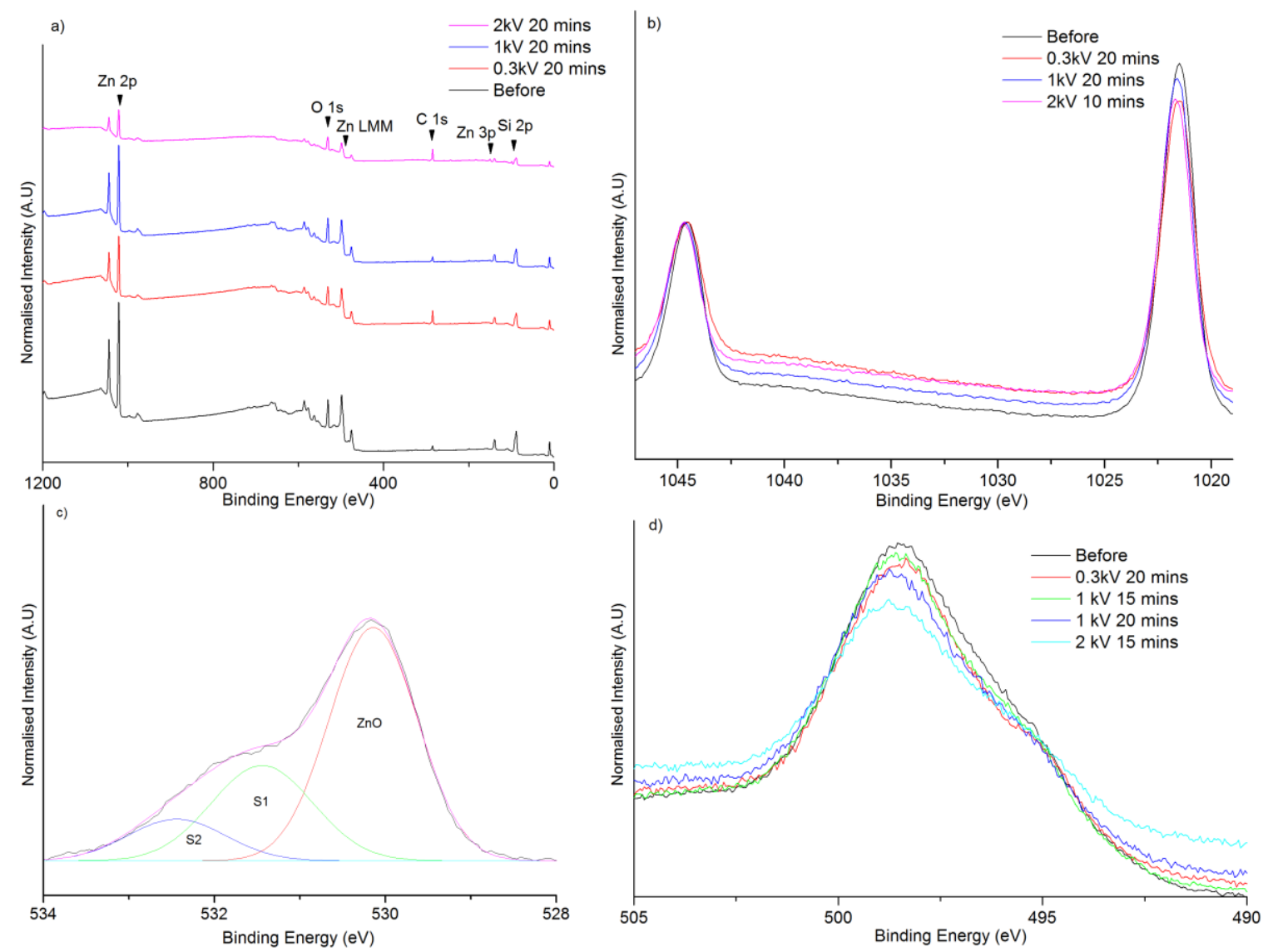

Figure 3. a) Evolution of the survey scan with argon bombardment dose, b) Evolution of the $\mathrm{Zn} 2 \mathrm{p}$ peak with argon bombardment, c) The $O$ 1s peak before argon bombardment, showing fits and background and d) Evolution Zn LMMA peak with argon bombardment.

There was no significant change in the $\mathrm{Zn} 3 \mathrm{p}$ or $2 \mathrm{p}$ peaks with argon bombardment; however, it is known these peaks are almost independent of the type of chemical bonds [17].

Before argon bombardment, the $\mathrm{O} 1 \mathrm{~s}$ peak consisted of a main component centred at 530.2 $\mathrm{eV}$, and a shoulder on the higher binding energy side consisting of two peaks offset from the main component by $1.35 \mathrm{eV}$ and $2.3 \mathrm{eV}$, labelled as $\mathrm{S} 1$ and $\mathrm{S} 2$, respectively. These peaks are attributed to lattice oxygen, surface hydroxide ions and surface water vapour, respectively [18-22]. Curve fitting area analysis of these peaks was used to calculate the percentage water vapour component contribution to the $\mathrm{O}$ 1s peak and the results are show in Figure 4a). Since the $\mathrm{C}$ 1s spectrum was not a suitable measure of surface contamination, the $\mathrm{S} 2$ component was used instead. The quantity of water measured decreased with both increased acceleration voltage and bombardment time; it was also noted that the S1 component followed similar trends. 

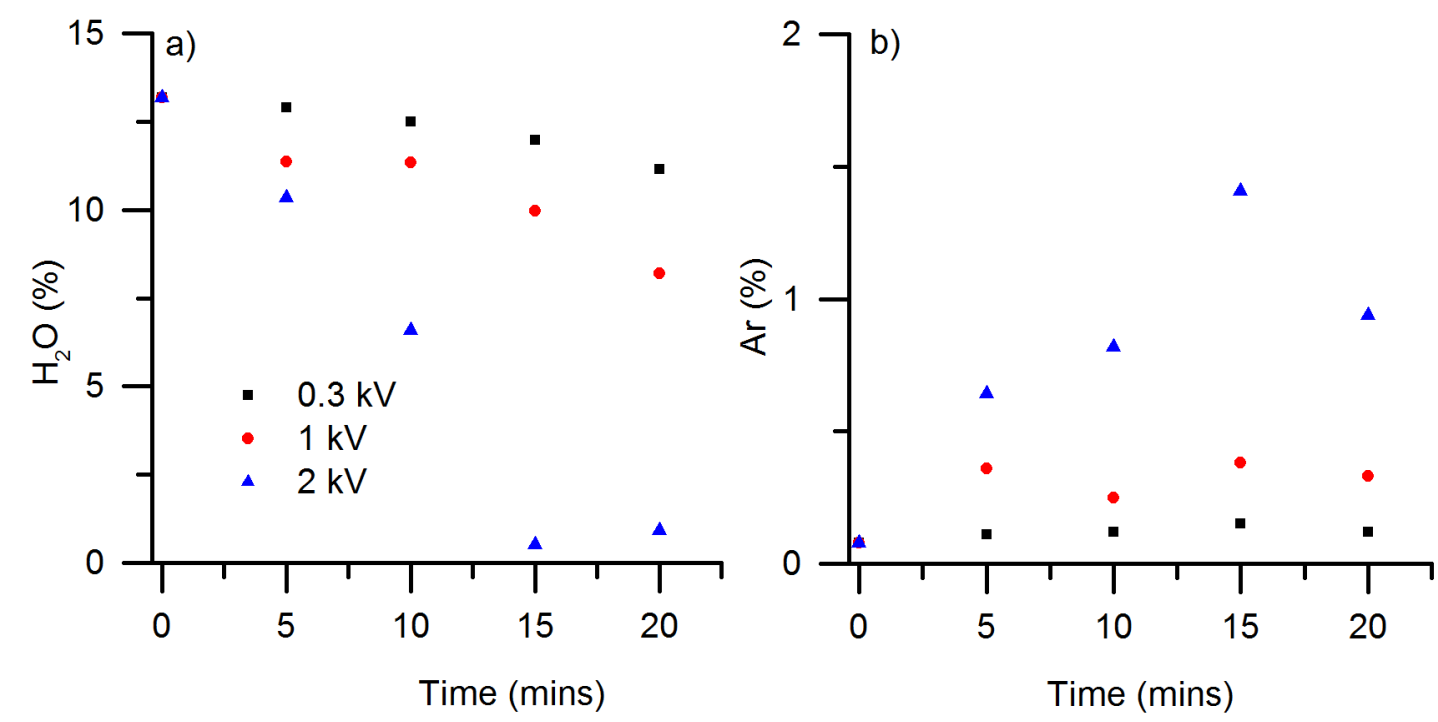

Figure 4: Plots of a) Percentage $\mathrm{H}_{2} \mathrm{O}$ and b) Argon against time of argon bombardment.

It is known that high energy ion bombardment can cause argon to be incorporated into the sample [23], and the Ar 2p spectra was therefore also fitted. Figure 4b) depicts the percentage Ar measured in each sample and shows that there was no increase in argon incorporated into the $\mathrm{ZnO}$ nanosheets by argon bombardment at $0.3 \mathrm{kV}$ as the quantity measured is not above the background noise of argon measured from the "before" sample. However, when an acceleration voltage of greater than $1 \mathrm{kV}$ was used, an increased quantity of argon was measured compared to the untreated sample.

The $\mathrm{Zn}$ LMMA peak, depicted in Figure 3d), is consistent with that of $\mathrm{ZnO}$ and did not alter shape with argon bombardment at $0.3 \mathrm{kV}$ and up to 15 mins at $1 \mathrm{kV}$. After the nanosheets were bombarded for 20 mins at $1 \mathrm{kV}$ and at $2 \mathrm{kV}$, the peak shape shift shows a peak centre at a lower binding energy; this change is consistent with the formation of metallic zinc, and is in agreement with the SEM images in Figure 2 which show damaged nanosheets at these acceleration voltages. 

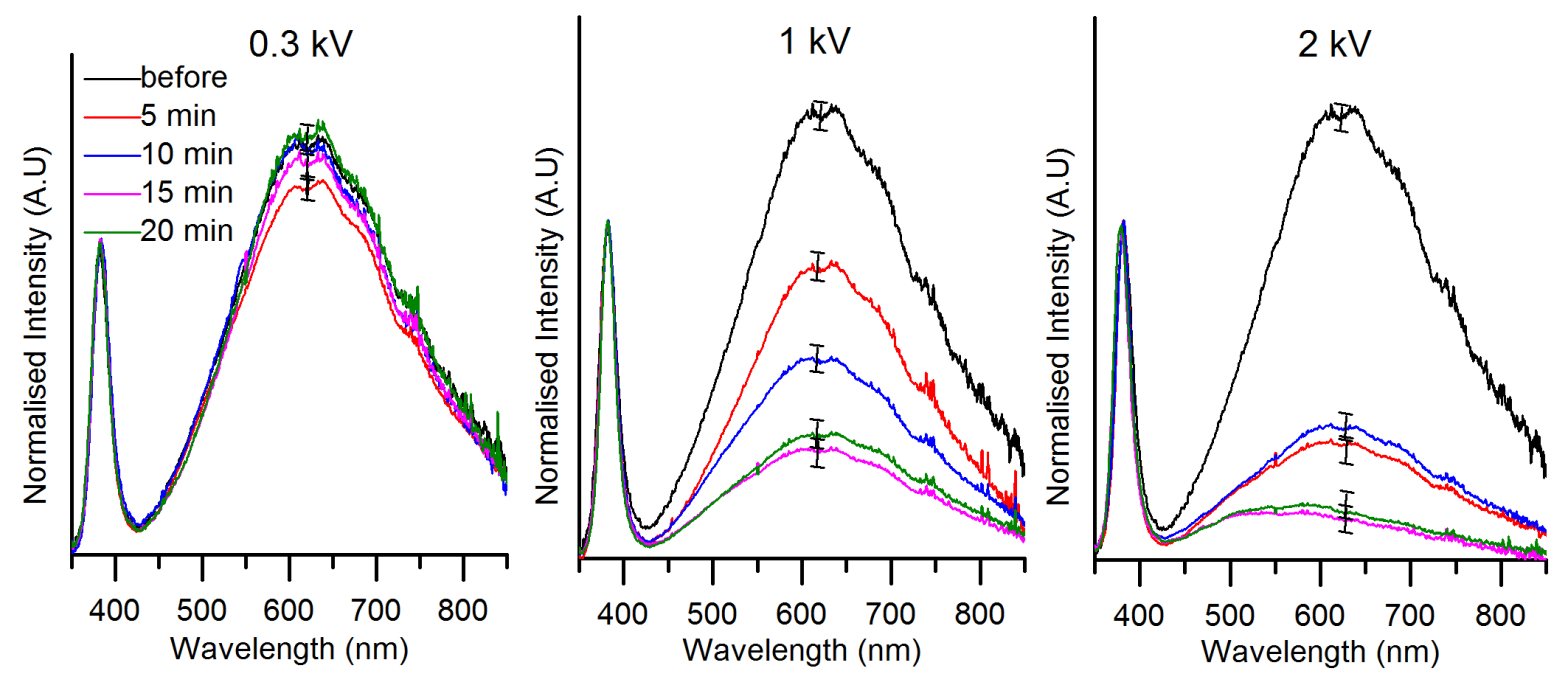

Figure 5: PL Spectra for $\mathrm{ZnO}$ nanosheets with different powers and time of argon bombardment. Error bar show standard deviation at $625 \mathrm{~nm}$.

PL was carried out to characterise changes in the defect chemistry with each spectrum normalised to the near band edge (NBE) peak, shown in Figure 5. Before argon bombardment, the NBE peak was centred at $381.3 \mathrm{~nm}$ and the deep level emission (DLE) peak in the range of $400-850 \mathrm{~nm}$ and shape of the DLE peaks is agreement with our previous work $[7,13]$. There has been great debate in the literature over the origins of the constituent components of the DLE peak and defects in $\mathrm{ZnO}$ as a whole. These debates centre around the role of zinc and oxygen vacancies and interstitials as well as hydrogen impurities and density of states due to confinement and how the nature of the defects affect the conduction mechanisms [24-37]. As there is no consensus on the exact position and contribution of these components, the following discussion will focus on the overall changes in relative intensity of the DLE peak.

Argon bombardment at $0.3 \mathrm{kV}$ did not alter the PL spectra, with both the NBE peak remaining centred at $381.3 \mathrm{~nm}$ for all time durations of bombardment. There were some variations in the relative intensities of the DLE peak but these were within the variation of the measurements and therefore not considered to be significant as the error bars overlap. Argon bombardment at $1 \mathrm{kV}$ also did not result in any measurable change to the position of the NBE peak. However, it was observed that the relative intensity of the DLE peak decreased with increased duration of bombardment. The spectra for the samples bombarded for 15 and 20 min do no statistically differ from each other as shown by the overlapping error bars. Our previous work [13] showed that using similar argon bombardment conditions stripped the 
surface of $\mathrm{ZnO}$ nanorods, and the SEM images in Figure 2 also indicate that the surface of the nanosheets are stripped. Since the normalised DLE peak reduced with argon bombardment duration, it suggests that the defects that cause the DLE peak are mainly situated at the near surface and are removed when the near surface is stripped.

Argon bombardment at $2 \mathrm{kV}$ for 5 and 10 minutes reduced the relative intensity of the DLE, with the unchanged peak shape and the position of the NBE peak did not shift during bombardment. Bombardment for 15 and 20 minutes further reduced the intensity of the DLE peak relative to the NBE peak, and again they did not significantly differ from each other. However as the Figure 2 shows, the nanosheets are damaged to a point where the surface is not clear therefore it is not possible to comment on the defect distribution for these samples. The position of the NBE peaks also shifted to $378.4 \mathrm{~nm}$.

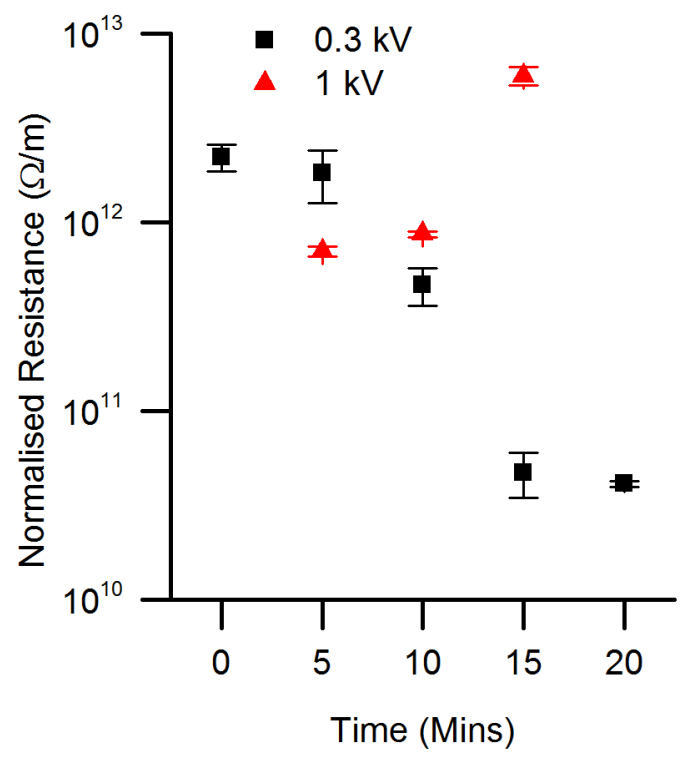

Figure 6. Graph of normalised resistance against duration of argon bombardment. Error bars show the standard error.

To assess the effects of argon bombardment on the transport properties of the $\mathrm{ZnO}$ nanosheets, nanoscale two-point probe was used to perform I-V measurements; the normalised resistance at $+1 \mathrm{~V}$ is plotted against argon bombardment duration in Figure 6 . Normalised resistance was calculated using the average of the measured resistances of the nanosheets divided by probe separation. Figure S2 in the supplemental information show SEM images of typical probe placements. The samples bombarded at $2 \mathrm{kV}$ and $1 \mathrm{kV}$ for 20 
minutes were not characterised as the SEM images showed damage to the nanostructures that would create inconsistent results.

The average normalised resistance before argon bombardment was $2.22 \times 10^{12} \Omega \mathrm{m}^{-1}$. Argon bombardment at $0.3 \mathrm{kV}$ for 20 mins caused a the normalised resistance to reduce to 4.12 $\times 10^{10} \Omega \mathrm{m}^{-1}$, although it should be noted that the difference between 15 minutes and 20 minutes is not statistically significant. These results are in agreement with our previous work [13], which attributed the effect to surface cleaning.

Argon bombardment at $1 \mathrm{kV}$ for 5 mins caused the normalised resistance to drop to $7.01 \times 10^{11} \Omega \mathrm{m}^{-1}$, however, further bombardment caused the resistance to increase, with the normalised resistance after 15 mins increasing to $5.96 \times 10^{12} \Omega \mathrm{m}^{-1}$. Again, this is in agreement with our previous work [13], which attributed this effect to the removal of defects resulting in a reduction in the number of charge carriers by stripping the surface of the nanostructure.

Figure 7 shows an I-V curve normalised to the current measured at $+2 \mathrm{~V}$ for a typical nanosheet after bombardment for 20mins at $0.3 \mathrm{kV}$ (“cleaned"), and bombarded at $1 \mathrm{kV}$ for 15 min ("stripped"). It was observed that the I-V curves measured before and after $0.3 \mathrm{kV}$ bombardment were slightly rectifying in character, indicating that the tungsten probes made a Schottky contact to the nanosheets. A linear fit was used to quantify a typical cleaned I-V curve and the $\mathrm{R}^{2}$ value was calculated to be 0.9917 . However, the stripped I-V curve was more rectifying in character and the $\mathrm{R}^{2}$ of a typical curve was calculated to be 0.9509 . This is in agreement with our work on $\mathrm{ZnO}$ [13], which showed that this effect is a result of surface charge being removed from the $\mathrm{ZnO}$ nanosheets by a reduction in donor states. Removal of these defects resulted in increased band bending at the boundary between the probe and the material surface, yielding a more rectifying contact. It should be noted that the reduction in the normalised DLE peak indicates that all defects have reduced. This includes acceptor/ptype defects as well as $\mathrm{n}$ type defects, however, it is known that $\mathrm{ZnO}$ is naturally $\mathrm{n}$-type therefore any reduction in acceptor defects has a minimal effect on the conduction mechanism when compared to a reduction in donor/ n-type defects [37]. It is noted that the contact made to the "cleaned" nanosheets is more rectifying than that made to "cleaned" nanorods in our previous work. We attribute this to the nanorods having a larger normalised defect peak, which was 2.5 times the intensity of the NBE peak, which corresponds to a 
higher concentration of donor states at the surface, in turn resulting in a lower degree of band bending at the interface with the tungsten probe.

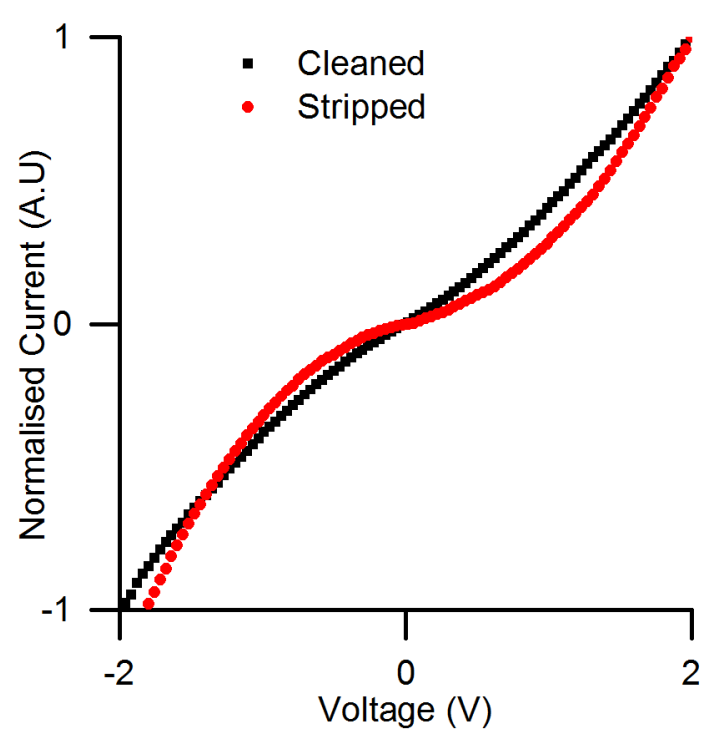

Figure 7. Graph of normalised current against voltage for a typical "cleaned" ZnO nanosheets and a typical "stripped" nanosheet.

\section{Conclusion}

$\mathrm{ZnO}$ nanosheets have been treated with increasing doses, by both power and time, of argon bombardment and the resulting changes in the electrical transport and defect chemistry have been measured experimentally using nanoscale two-point probe, XPS, PL and SEM. Lowpowered, $0.3 \mathrm{kV}$ doses of argon bombardment removed surface contamination without changing the defect chemistry. It was found that at least 15 minutes of treatment was needed to remove surface contamination and to reduce the resistance measured to a minimum, two orders of magnitude lower than before treatment.

Higher-energy bombardment at $1 \mathrm{kV}$ resulted in surface stripping of the $\mathrm{ZnO}$ nanosheets, with 20 mins of bombardment causing holes to appear in the nanosheet structures. As the surface was stripped, the PL defect concentration reduced and the measured resistance increased. The reduction in defects resulted in less surface charge due to the removal of donor states causing increased band bending at the contact. Argon bombardment at $2 \mathrm{kV}$ caused significant damage to the nanosheets, making it impossible to carry out reliable conductivity measurements. 
The work presented herein indicates that removing surface contamination with a low-energy dose of argon bombardment results in more reproducible, lower resistance contacts without altering the defect chemistry. Our work also shows that the contact type can be controlled by selectively surface-stripping $\mathrm{ZnO}$ nanosheets with higher-energy doses of argon bombardment. The controlled removal of the defects and the ability to control the conduction/donor concentration of the nanostructure allows for enhancement of the sensitivity of devices such as gas sensors [4] and more efficient solar cells [6]. The work also indicates a possibility of controlling doping concentration within layers of the same nanostructure without the need for dopant implantation, which may be achieved by exposing only one side of the nanostructure to bombardment.

The results presented in this investigation agree with our previous work on $\mathrm{ZnO}$ nanorods. Within that study, it was found that defect states are concentrated in the surface of nanostructures even if they are polycrystalline in nature. It should be noted that the polycrystalline structure of $\mathrm{ZnO}$ nanosheets is more susceptible to damage from argon bombardment than single crystal $\mathrm{ZnO}$ nanorods, and that nanosheets appear to have a lower concentration of defects, which can result in a more rectifying contact.

\section{ACKNOWLEDGMENTS}

The authors gratefully acknowledge the financial support provided by the Welsh Government Sêr Cymru National Research Network in Advanced Engineering and Materials (NRN-150 and NRN-123)

\section{References}

[1] Cheng $\mathrm{H}$, Cheng J, Zhang $\mathrm{Y}$ and Wang Q-M 2007 Large-scale fabrication of ZnO micro-and nano-structures by microwave thermal evaporation deposition Journal of Crystal Growth 299 34-40

[2] Schmidt-Mende L and MacManus-Driscoll J L 2007 ZnO - nanostructures, defects, and devices Materials Today 10 40-8

[3] Tarat A, Majithia R, Brown R A, Penny M W, Meissner K E and Maffeis T G G 2012 Nanocrystalline $\mathrm{ZnO}$ obtained from pyrolytic decomposition of layered basic zinc acetate: Comparison between conventional and microwave oven growth20-23 Aug. 2012) p 1-5

[4] Jones D R and Maffeïs T G G 2015 Analysis of the kinetics of surface reactions on a zinc oxide nanosheet-based carbon monoxide sensor using an Eley-Rideal model Sensors and Actuators B: Chemical 218 16-24

[5] Tarat A, Majithia R, Brown R, Penny M and Meissner K 2012 Synthesis of nanocrystalline ZnO nanobelts via pyrolytic decomposition of zinc acetate nanobelts and their gas sensing behavior Surf Sci 606715 - 21 
[6] Tarat A, Nettle C, Bryant D T J, Jones D, Penny M, Brown R, Majitha R, Meissner K and Maffeis T G G 2014 Microwave-assisted synthesis of layered basic zinc acetate nanosheets and their thermal decomposition into nanocrystalline ZnO Nanoscale Research Letters 911

[7] Barnett C, Smith N, Jones D, Maffeis T G and Cobley R 2015 Effects of Vacuum Annealing on the Conduction Characteristics of ZnO Nanosheets Nanoscale Res. Lett. 10 1-6

[8] Law J B K and Thong J T L 2008 Improving the NH 3 gas sensitivity of ZnO nanowire sensors by reducing the carrier concentration Nanotechnology 19205502

[9] Ra H W, Khan R, Kim J T, Kang B R, Bai K H and Im Y H 2009 Effects of surface modification of the individual $\mathrm{ZnO}$ nanowire with oxygen plasma treatment Materials Letters $632516-9$

[10] Ra H-W and Im Y-H 2008 Effect of chemically reactive species on properties of ZnO nanowires exposed to oxygen and hydrogen plasma Nanotechnology 19485710

[11] Ra H-W, Choi K S, Ok C W, Jo S Y, Bai K H and Im Y H 2008 lon bombardment effects on ZnO nanowires during plasma treatment APPLIED PHYSICS LETTERS 93 -

[12] Dulub O, Boatner L A and Diebold U 2002 STM study of the geometric and electronic structure of $\mathrm{ZnO}(0001)-\mathrm{Zn},(000-1)-0,(10-10)$, and (11-20) surfaces Surf. Sci. 519 201-17

[13] Barnett C J, Kryvchenkova O, Smith N A, Kelleher L, Maffeis T G G and Cobley R J 2015 The effects of surface stripping ZnO nanorods with argon bombardment Nanotechnol. 26415701

[14] Barnett C J, Kryvchenkova O, Wilson L S J, Maffeis T G G, Kalna K and Cobley R J 2015 The role of probe oxide in local surface conductivity measurements J. Appl. Phys. 117174306

[15] Cobley R J, Brown R A, Barnett C J, Maffeis T G G and Penny M W 2013 Quantitative analysis of annealed scanning probe tips using energy dispersive x-ray spectroscopy Appl. Phys. Lett. 102

[16] Smith N A, Lord A M, Evans J E, Barnett C J, Cobley R J and Wilks S P 2015 Forming reproducible non-lithographic nanocontacts to assess the effect of contact compressive strain in nanomaterials Semicond. Sci. Technol.

[17] Gao Y K, Traeger F, Shekhah O, Idriss H and Wöll C 2009 Probing the interaction of the amino acid alanine with the surface of ZnO Journal of Colloid and Interface Science 338 16-21

[18] Heinhold R, Cooil S P, Evans D A and Allen M W 2014 Stability of the Surface Electron Accumulation Layers on the Nonpolar (1010) and (1120) Faces of ZnO The Journal of Physical Chemistry C 118 24575-82

[19] Byrne D, McGlynn E, Henry M O, Kumar K and Hughes G 2010 A novel, substrate independent three-step process for the growth of uniform $\mathrm{ZnO}$ nanorod arrays Thin Solid Films 518 4489-92

[20] Crist B V 2005 PDF Handbooks of Monochromatic XPS Spectra Volume 2 Pure Binary Oxides

[21] Maffeis T G G, Penny M W, Castaing A, Guy O J and Wilks S P 2012 XPS investigation of vacuum annealed vertically aligned ultralong ZnO nanowires Surf. Sci. 606 99-103

[22] Barnett C J, Castaing A, Jones D R, R. L A, Jenkins L J, Cobley R J and Maffeis T G G 2017 XPS investigation of titanium contact formation to ZnO nanowires Nanotechnology 28085301

[23] Lau W M, Bello I, Huang L J, Feng X, Vos M and Mitchell I V 1993 Argon incorporation in Si(100) by ion bombardment at 15-100 eV J. Appl. Phys. 74 7101-6

[24] Wang M, Zhou Y, Zhang Y, Jung Kim E, Hong Hahn S and Gie Seong S 2012 Near-infrared photoluminescence from ZnO APPLIED PHYSICS LETTERS 100101906

[25] Xu C, Chun J and Kimb D E 2007 Electrical properties and near band edge emission of Bidoped ZnO nanowires APPLIED PHYSICS LETTERS 90 1-3

[26] Yang Y, Yan H, Fu Z, Yang B, Xia L, Xu Y, Zuo J and Li F 2006 Photoluminescence and Raman studies of electrochemically as-grown and annealed $\mathrm{ZnO}$ films Solid State Communications 138 521-5

[27] Lima S A M, Sigoli F A, Jafelicci Jr M and Davolos M R 2001 Luminescent properties and lattice defects correlation on zinc oxide International Journal of Inorganic Materials 3 749-54

[28] Lin B, Fu Z and Jia Y 2001 Green luminescent center in undoped zinc oxide films deposited on silicon substrates APPLIED PHYSICS LETTERS 79 943-5 
[29] Liao Z-M, Zhang H-Z, Zhou Y-B, Xu J, Zhang J-M and Yu D-P 2008 Surface effects on photoluminescence of single ZnO nanowires Physics Letters A 372 4505-9

[30] Heo Y W, Norton D P and Pearton S J 2005 Origin of green luminescence in ZnO thin film grown by molecular-beam epitaxy Journal of Applied Physics 98073502

[31] Janotti $A$ and Van de Walle C G 2005 Oxygen vacancies in ZnO APPLIED PHYSICS LETTERS 87 -

[32] Wei X Q, Man B Y, Liu M, Xue C S, Zhuang H Z and Yang C 2007 Blue luminescent centers and microstructural evaluation by XPS and Raman in $\mathrm{ZnO}$ thin films annealed in vacuum, N2 and O2 Physica B: Condensed Matter 388 145-52

[33] Xu P S, Sun Y M, Shi C S, Xu F Q and Pan H B 2003 The electronic structure and spectral properties of $\mathrm{ZnO}$ and its defects Nuclear Instruments and Methods in Physics Research Section B: Beam Interactions with Materials and Atoms 199 286-90

[34] Lijie L 2017 On the double-band luminescence of ZnO nanoparticles EPL (Europhysics Letters) 11767005

[35] Janotti A and Van de Walle C G 2007 Native point defects in ZnO Physical Review B 76 165202

[36] Fumiyasu O, Minseok C, Atsushi T and Isao T 2011 Point defects in ZnO: an approach from first principles Science and Technology of Advanced Materials 12034302

[37] McCluskey M D and Jokela S J 2009 Defects in ZnO Journal of Applied Physics 106 - 